\title{
Therapy Line and Associated Predictors of Response to PD-1/ PD-L1-Inhibitor Monotherapy in Advanced Non-small-Cell Lung Cancer: A Retrospective Bi-centric Cohort Study
}

\author{
David Lang ${ }^{1} \mathbb{0}$ - Florian Huemer ${ }^{2} \cdot$ Gabriel Rinnerthaler $^{2} \cdot$ Andreas Horner $^{1} \cdot$ Romana Wass $^{1} \cdot$ Elmar Brehm $^{1}$. \\ Kaveh Akbari ${ }^{3} \cdot$ Marcel Granitz ${ }^{4} \cdot$ Georg Hutarew $^{5} \cdot$ Bernhard Kaiser $^{1} \cdot$ Richard Greil $^{2} \cdot$ Bernd Lamprecht $^{1}$
}

Published online: 25 October 2019

(c) The Author(s) 2019

\begin{abstract}
Background Evidence on PD-1/PD-L1-directed immune checkpoint inhibitor (ICI) therapy for advanced non-small-cell lung cancer (NSCLC) is mainly based on clinical trials in first- or second-line settings.

Objective We aimed to investigate response and prognostic factors with special regard to third- or later-line therapy.

Patients and Methods We retrospectively analyzed all patients who had received ICI monotherapy with nivolumab, pembrolizumab, or atezolizumab for advanced NSCLC. Computed tomography evaluations were analyzed using response evaluation criteria in solid tumors (RECIST, version 1.1). Kaplan-Meier analyses were conducted to calculate progression-free (PFS) and overall (OS) survival; the impact of influencing variables was evaluated using uni- and multivariate Cox-regression analyses.

Results Among 153 patients (59\% men, mean age 66 years), median PFS was 4 months [mo; 95\% confidence interval (95\% CI) 3-5], OS was 13 mo (10-17), and objective response rate (ORR) was $22 \%$. Therapy line $\geq 3$ was associated with significantly inferior PFS $(p=0.003)$ and OS ( $p=0.001)$. In first-line therapy PFS, OS, and ORR were 7 mo (3-11), 17 mo [9-not evaluable (n.e.)], and 36\%; in second-line 4 mo (3-7), 18 mo (13-n.e.) and 19\%, and in $\geq$ third-line 2 mo (1-3), 9 mo (4-12), and $13 \%$. PFS was significantly influenced by PD-L1 expression in first-line therapy $(p=0.006)$. In $\geq$ third-line patients, Eastern Cooperative Oncology Group (ECOG) performance status significantly affected PFS and OS (both $p<0.001$ ).

Conclusions Third- or later-line single-agent anti-PD-1/PD-L1 therapy is less efficacious as compared to first- and secondline treatment. In that setting, ECOG performance status predominates known predictors like PD-L1 expression or presence of an alteration in EGFR or ALK.
\end{abstract}

\section{Introduction}

Inhibition of programmed death-ligand 1 (PD-L1) or programmed cell death protein 1 (PD-1) has fundamentally changed lung cancer therapy [1]. Currently, literally any patient with advanced non-small-cell lung cancer (NSCLC) without contraindications will-given a sufficiently long survival period-receive immune checkpoint inhibitor (ICI) treatment at some point during the course of disease. Still, only a minority of patients actually benefit from ICI monotherapy, and foreseeing the individual patient's response is difficult [2].

David Lang

lunge@kepleruniklinikum.at;

david.lang@kepleruniklinikum.at

Extended author information available on the last page of the article
Since the advent of ICI therapies for NSCLC, ICI monotherapy has been widely applied, especially in second-line settings following progression after first-line chemotherapy $[1,3-6]$ or in first-line therapy for highly PD-L1-expressing tumors [7]. Recently, combinations of ICI and platinumbased doublet chemotherapy have been established as the first-line standard for stage IV NSCLC [8, 9]. Also, quadruple combinations including bevacizumab have entered daily clinical practice, especially in EGFR (epidermal growth factor receptor) or ALK (anaplastic lymphoma kinase) mutant patients with no more options for tyrosine kinase inhibitor (TKI) therapy [10]. The value of ICI/ICI combination therapy, however, has not yet been fully clarified, but data are promising [11].

Despite these rapidly evolving combination regimens, a considerable percentage of patients will still receive ICI monotherapy, especially heavily pretreated patients in 


\section{Key Points}

Compared to first- or second-line of treatment, NSCLC patients who had received PD-1/PD-L1-directed immune checkpoint inhibitor monotherapy in line $\geq$ three had significantly inferior PFS and OS.

In multivariate analyses among all patients, PFS was significantly influenced by PD-L1 expression and ECOG performance status, for OS only therapy line was shown to have a significant impact.

In first-line treated patients, PFS was significantly influenced by PD-L1 expression, while there were no significant multivariate findings for first-line OS and secondline PFS/OS. In $\geq$ third-line patients, ECOG performance status significantly affected PFS and OS.

third- or later-line settings who have not received ICI therapy before. Patients in these situations are usually characterized by lower performance status, co-morbidities, and treatmentrelated toxicities restricting available treatment options. Evidence on known predictors of response like PD-L1 expression, EGFR mutational status, or tumor mutational burden (TMB) is mainly derived from first- or second-line therapy studies [7, 11, 12]. Thus, it appears questionable if those biomarkers have the same prognostic and/or predictive properties in third- or later-line therapy settings.

Our aim was to evaluate whether response to PD-1/PD-L1 ICI monotherapy changes with therapy line and which patient or tumor-related factors are associated with patient outcomes in a real-life setting.

\section{Patients and Methods}

One hundred and fifty-three consecutive patients with advanced NSCLC who had received at least one cycle of nivolumab, pembrolizumab, or atezolizumab at the lung cancer unit of Kepler University Hospital Linz and the medical oncology unit of Paracelsus Medical University Salzburg between May 2015 and June 2018 were retrospectively registered.

First-line therapy was defined as primary treatment in a non-curable (e.g., stage IV [13] or not otherwise treatable stage III) setting, not considering previous therapies in potentially curable stages. Patients in stage III disease were eligible to receive ICI therapy and to be included in the study if they were pre-treated by chemo(radio)therapy and another line of chemotherapy was not reasonably feasible. Also, in selected cases upon contraindications to chemotherapy and despite PD-L $1<50 \%$, a first-line ICI treatment in stage
IV disease could be applied after multidisciplinary tumorboard discussion. We excluded patients in clinical trials, on ICI/chemotherapy or ICI/ICI combinations, and those who received ICI for thoracic malignancies other than NSCLC.

Patients were retrospectively followed from ICI therapy initiation to death or censored at the date of the last verified contact. The time of disease progression was retrospectively defined by imaging and death, as well as by the patients' medical records. In selected cases of considerable clinical benefit, ICI therapy could be applied beyond the determined point of disease progression. Radiological response was routinely assessed by an iodinated contrast mediumenhanced CT scan of the chest and the upper abdomen after four cycles of nivolumab or three cycles of pembrolizumab/ atezolizumab, equaling a time interval of 10 or 12 weeks, respectively. Re-staging could be preponed in case of clinical suspicion of disease progression, and additional/alternative imaging modalities like ${ }^{18} \mathrm{~F}$-FDG-PET/CT or cerebral magnetic resonance tomography could be applied if necessary, according to the clinician's judgment. For this study, radiological response was re-evaluated by an expert thoracic radiologist and graded by Response Evaluation Criteria in Solid Tumors (RECIST), version 1.1 [14] for first and best response ( $\mathrm{CR}$ - complete remission, $\mathrm{PR}$ - partial remission, SD—stable disease, PD—progressive disease). Overall response rate (ORR) was defined as the percentage of patients having reached a best response of CR or PR.

Kaplan-Meier-analyses for PFS and OS were conducted for all patients and according to therapy line (first line, second line, $\geq$ third line). Results were expressed as median in months [95\% confidence interval (CI)], unless otherwise specified. The Kaplan-Meier survival curves were compared statistically using the log rank test, and a $p$ value $<0.05$ was regarded as statistically significant.

Evaluation of predictive factors for PFS and OS was conducted applying uni- and multivariate Cox-regression analyses. Variables analyzed in these models were age groups ( $</ \geq 70$ years), sex, histological subtype (adeno-, squamous-cell carcinoma), presence of brain metastases, palliative therapy line (1 vs. 2, $\geq 3$ ), ECOG (Eastern Cooperative Oncology Group) performance status (0 vs. 1, 2, 3) and presence of a targetable genetic tumor alteration (ALK, EGFR, ROS). PD-L1 expression on tumor cells was assessed with a 22C3 assay for Autostainer Link 48 by Dako (Agilent Technologies, Santa Clara, CA, USA), whereas a negative PD-L1 status was defined as a proportion of $<1 \%$ of viable tumor cells showing membranous staining.

\section{Results}

Baseline patient and tumor characteristics for all patients and according to therapy line are shown in Table 1. 
Table 1 Baseline patient and tumor characteristics for all patients and according to therapy line

\begin{tabular}{|c|c|c|c|c|}
\hline & $\begin{array}{l}\text { All patients } \\
(N=153)\end{array}$ & $\begin{array}{l}\text { First-line therapy } \\
(N=45)\end{array}$ & $\begin{array}{l}\text { Second-line therapy } \\
(N=70)\end{array}$ & $\begin{array}{l}\geq \text { Third-line therapy } \\
(N=38)\end{array}$ \\
\hline Age (mean, SD) & $66(11)$ & $72(10)$ & $66(9)$ & $61(12)$ \\
\hline Age range (years) & $26-90$ & $47-90$ & $39-85$ & $26-81$ \\
\hline \multicolumn{5}{|l|}{ ECOG status } \\
\hline 0 & $45(29)$ & $16(36)$ & $17(24)$ & $12(29)$ \\
\hline 1 & $91(59)$ & $27(60)$ & $44(63)$ & $20(60)$ \\
\hline 2 & $14(10)$ & $1(2)$ & $9(13)$ & $4(9)$ \\
\hline 3 & $3(2)$ & $1(2)$ & 0 & $2(2)$ \\
\hline \multicolumn{5}{|l|}{ Sex } \\
\hline Female & $62(41)$ & $19(42)$ & $28(40)$ & $15(40)$ \\
\hline Male & $91(59)$ & $26(58)$ & $42(60)$ & $23(60)$ \\
\hline \multicolumn{5}{|l|}{ ICI substance } \\
\hline Nivolumab & $80(52)$ & $16(36)$ & $37(53)$ & $27(71)$ \\
\hline Pembrolizumab & $58(38)$ & $26(58)$ & $26(37)$ & $6(16)$ \\
\hline Atezolizumab & $15(10)$ & $3(7)$ & $7(10)$ & $5(13)$ \\
\hline \multicolumn{5}{|l|}{ Smoking status } \\
\hline Never/ $\leq 5$ py & $20(13)$ & $5(11)$ & $8(11)$ & $7(18)$ \\
\hline$>5$ py & $123(80)$ & $40(89)$ & $56(80)$ & $27(71)$ \\
\hline Unknown & $10(7)$ & & $6(9)$ & $4(11)$ \\
\hline Total py (mean, SD) & $44(34)$ & $43(25)$ & $48(39)$ & $38(35)$ \\
\hline \multicolumn{5}{|l|}{ Histology } \\
\hline Adenocarcinoma & $100(65)$ & $19(42)$ & $49(79)$ & $32(84)$ \\
\hline Squamous cell carcinoma & $53(35)$ & $26(58)$ & $21(30)$ & $6(16)$ \\
\hline \multicolumn{5}{|l|}{ TNM stage } \\
\hline III & $19(12)$ & $14(31)$ & $3(4)$ & $2(5)$ \\
\hline IV & $134(88)$ & $31(69)$ & $67(96)$ & $36(95)$ \\
\hline CNS involvement & $31(20)$ & $4(9)$ & $16(23)$ & $11(29)$ \\
\hline \multicolumn{5}{|l|}{ Genetic alteration } \\
\hline EGFR & $13(8)$ & & $4(6)$ & $9(24)$ \\
\hline ALK & $2(1)$ & & & $2(5)$ \\
\hline ROS1 & $3(2)$ & & $3(4)$ & \\
\hline \multicolumn{5}{|l|}{ PD-L1 status } \\
\hline Not available & $21(14)$ & $2(4)$ & $10(14)$ & $9(24)$ \\
\hline Positive & $85(56)$ & $30(67)$ & $42(60)$ & $13(34)$ \\
\hline Negative & $47(31)$ & $13(29)$ & $18(26)$ & $16(42)$ \\
\hline \multicolumn{5}{|l|}{ PD-L1 expression } \\
\hline Not available & $24(16)$ & $3(7)$ & $12(17)$ & $9(24)$ \\
\hline$<1 \%$ & $47(31)$ & $13(29)$ & $18(26)$ & $16(42)$ \\
\hline $1-49 \%$ & $44(29)$ & $13(29)$ & $24(34)$ & $7(18)$ \\
\hline$\geq 50 \%$ & $38(25)$ & $16(36)$ & $16(23)$ & $6(16)$ \\
\hline
\end{tabular}

Figures are given as absolute number and percent within the respective group unless otherwise specified. The numeric discrepancies between PD-L1 status and PD-L1 expression are due to patients with pathologically determined positive PD-L1 status but without exact quantification being reported or with further quantification being impossible $(n=3)$

$S D$ standard deviation, ECOG Eastern Cooperative Oncology Group, ICI immune checkpoint inhibitor, py pack years, TNM TNM Classification of Malignant Tumours, CNS central nervous system, EGFR epidermal growth factor receptor, ALK Anaplastic Lymphoma Kinase, ROS1 protooncogene tyrosine-protein kinase ROS, $P D-L 1$ Programmed Death-Ligand 1

PFS and OS as well as radiological response in all patients and according to the respective therapy line are shown in Table 2. Kaplan-Meier analyses (Fig. 1) showed that therapy line significantly influenced PFS $(p=0.003)$ and OS $(p=0.001)$. A univariate Cox-regression analysis confirmed that patients in $\geq$ third-line treatment had a 
Table 2 Radiological first and best response, objective response rate, progression-free and overall survival in all patients and according to therapy line

\begin{tabular}{|c|c|c|c|c|}
\hline & $\begin{array}{l}\text { All patients } \\
(N=153)\end{array}$ & $\begin{array}{l}\text { First-line therapy } \\
(N=45)\end{array}$ & $\begin{array}{l}\text { Second-line therapy } \\
(N=70)\end{array}$ & $\begin{array}{l}\geq \text { Third-line therapy } \\
(N=38)\end{array}$ \\
\hline \multicolumn{5}{|l|}{ First response (RECIST) } \\
\hline Not available & $34(22)$ & $3(7)$ & $25(36)$ & $6(16)$ \\
\hline Complete remission & $1(1)$ & & $1(1)$ & \\
\hline Partial remission & $25(16)$ & $12(27)$ & $9(13)$ & $4(11)$ \\
\hline Stable disease & $40(26)$ & $15(33)$ & $14(20)$ & $11(29)$ \\
\hline Progressive disease & $53(35)$ & $15(33)$ & $21(30)$ & $17(45)$ \\
\hline \multicolumn{5}{|l|}{ Best response (RECIST) } \\
\hline Not available & $34(22)$ & $3(7)$ & $25(36)$ & $6(16)$ \\
\hline Complete remission & $3(2)$ & $1(2)$ & $1(1)$ & $1(3)$ \\
\hline Partial remission & $31(20)$ & $15(33)$ & $12(17)$ & $4(11)$ \\
\hline Stable disease & $33(22)$ & $11(24)$ & $13(19)$ & $9(24)$ \\
\hline Progressive disease & $52(34)$ & $15(33)$ & $19(27)$ & $18(47)$ \\
\hline Objective response (rate in \%) & $34(22)$ & $16(36)$ & $13(19)$ & $5(13)$ \\
\hline Median progression-free survival (95\% CI) & $4(3,5)$ & $7(3,11)$ & $4(3,7)$ & $2(1,3)$ \\
\hline Median overall survival $(95 \% \mathrm{CI})$ & $13(10,17)$ & $17(9,-)$ & $18(13,-)$ & $9(4,12)$ \\
\hline
\end{tabular}

Figures are given as absolute number and percent within the respective group unless otherwise specified. Objective response rate includes patients with a RECIST best response of complete or partial remission

RECIST Response Evaluation Criteria in Solid Tumors, CI confidence interval

significant disadvantage concerning PFS $(p=0.005)$ and OS $(p=0.002)$. For patients in $\geq$ third-line therapy, hazard ratio (HR) was $1.97(1.20-3.21 ; p=0.007)$ for PFS and $1.99(1.12-3.53 ; p=0.019)$ for OS as compared to first-line therapy. Second-line compared to first-line therapy did not pose a significant risk for inferior PFS [HR $1.02(0.65-1.60$; $p=0.941)$ ] or OS [HR $0.82(0.46-1.46 ; p=0.493)]$.

In the whole patient cohort, the multivariate model (Table 3) revealed that PFS was significantly influenced by PD-L1 status $(p=0.002)$ and ECOG performance status $(p=0.029)$. For OS, the only significant variable identified was therapy line $(p=0.025)$.

For first-line therapy patients, the multivariate Cox-regression analysis revealed a negative PD-L1 status as significant predictor of PFS $(p=0.006)$, while for OS no variable was significant. In the second-line setting, no variable had significant impact on either PFS or OS. In third-line therapy, both PFS and OS (both $p<0.001$ ) were significantly determined by ECOG performance status. A significant signal for inferior OS in squamous-cell carcinoma patients in the univariate analysis could not be re-enacted in the multivariate evaluation (Table 4).

\section{Discussion}

Our data suggest that patients receiving single-agent PD-1/ PD-L1 ICI therapy in $\geq$ third-line therapy have significantly inferior PFS and OS compared to first- or second-line treatment. While "traditional" predictive factors like PD-L1 expression are relevant for PFS in first-line treated patients, they seem to have less impact in third- or later-line-treated patients, where only ECOG performance status had significant implications on PFS and OS.

Most phase 3 clinical trials that finally led to the approval of nivolumab, pembrolizumab, and atezolizumab were open for patients with more than one prior line of therapy. As an exception, CheckMate-057 only included second-line patients and CheckMate-017 only allowed for additional TKI therapy or switch maintenance. All those studies had an emphasis on second-line patients, as shown in Table 5 [3-6, 15-20]. Generally, ORR, PFS, and OS decreased with increasing number of previous therapy lines, which is consistent with our findings. Reported outcomes concerning PFS and OS tend to partly surpass our results; however, this may reflect the difference between a real-life cohort and a clinical trial setting. Of note, the mentioned trials included, with very few exceptions, only patients with ECOG performance status 0 or 1 . On the contrary, our reported collective comprised 10\% of ECOG 2 and 2\% of ECOG 3 patients. Thus, an OS of 9 months in a $\geq$ third-line setting appears to be a very promising result, while the comparatively short PFS of only 2 months requires further considerations: The natural course of disease in a third-line therapy stage IV NSCLC setting has itself never been studied to our knowledge. There was, however, a placebo arm in a study by Shepherd et al. (2005) evaluating erlotinib in chemotherapy-pretreated 


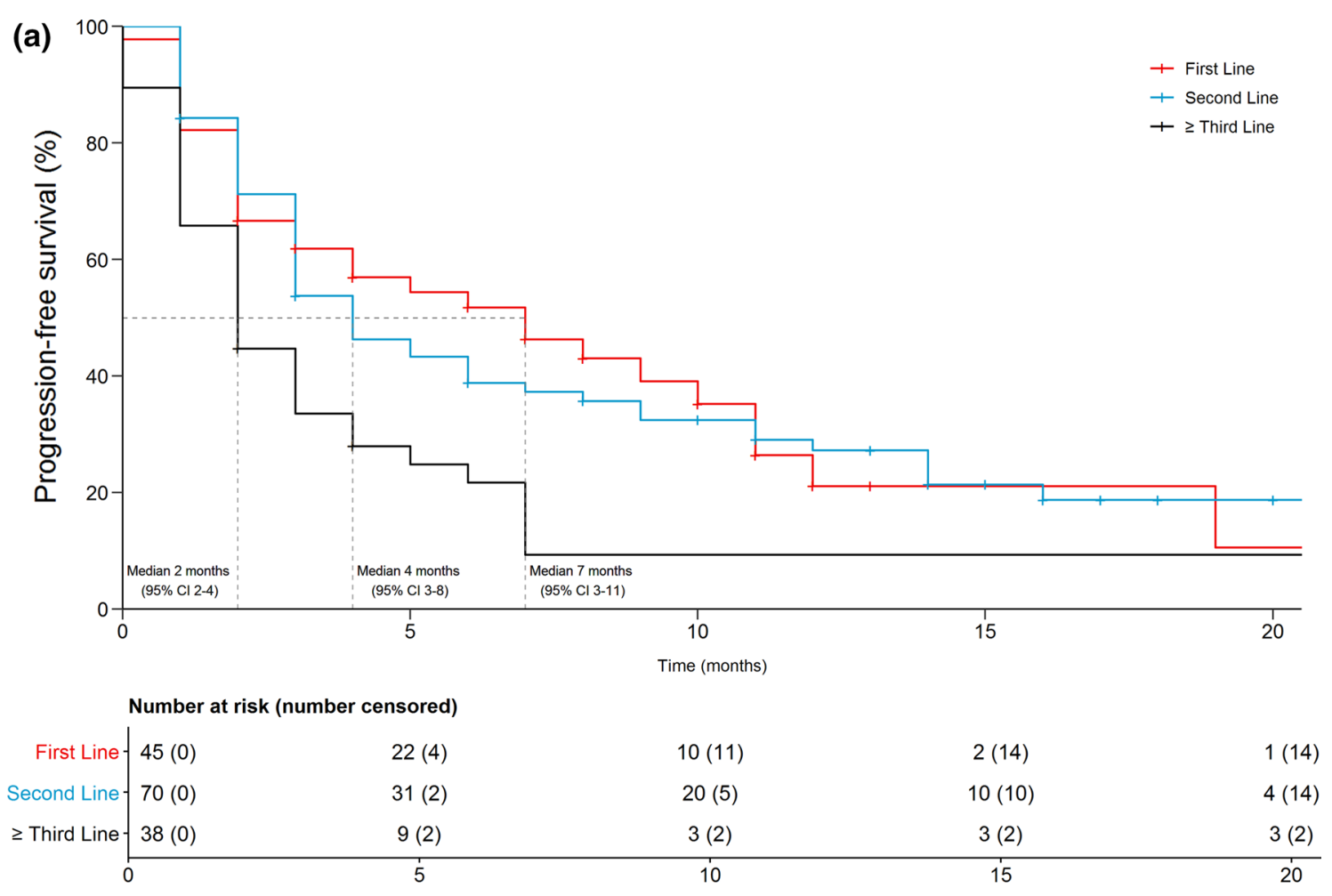

(b)

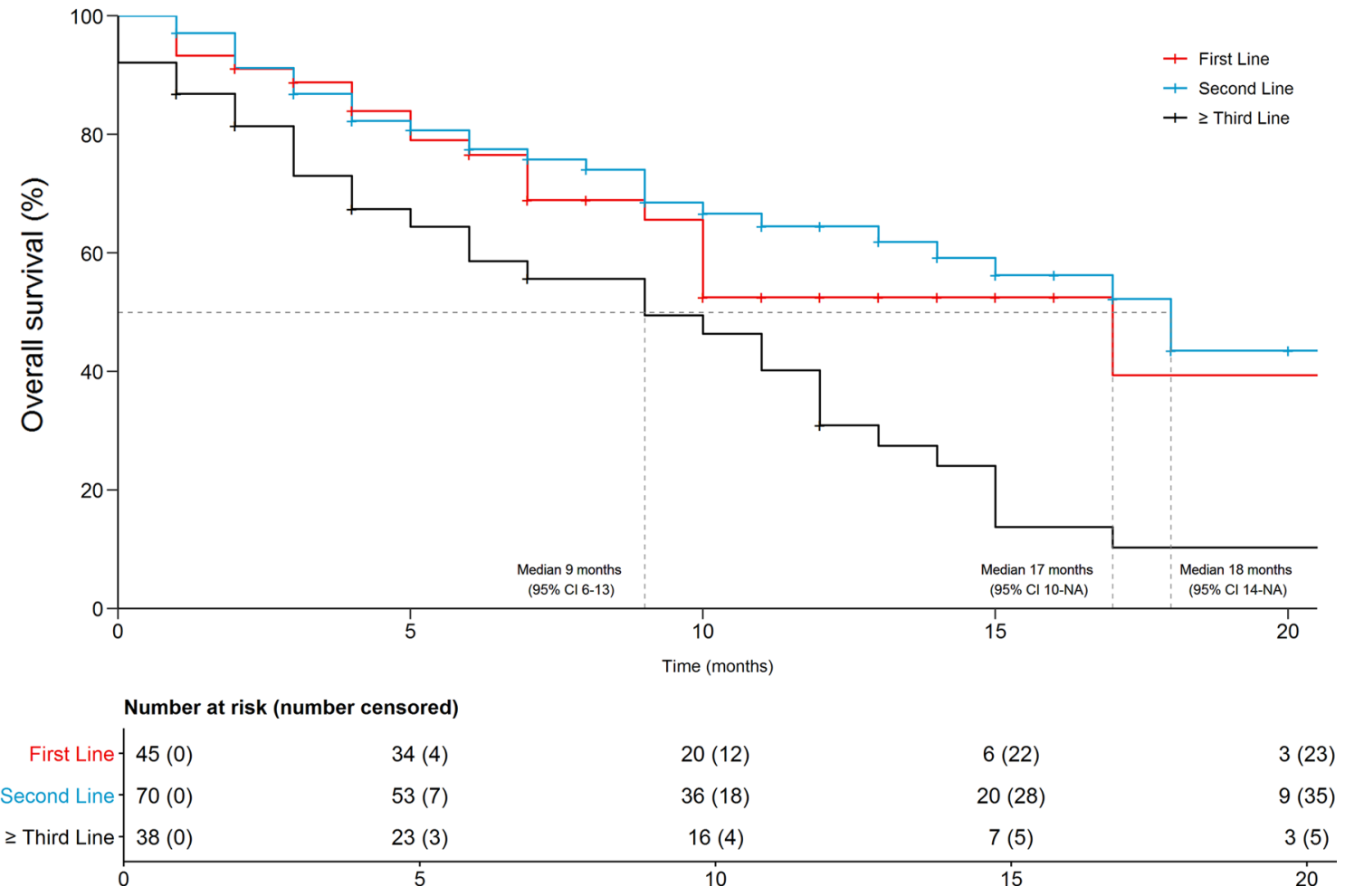

Fig. 1 Kaplan-Meier curves for progression-free (a) and overall (b) survival according to therapy line. NA not available, $C I$ confidence interval 
Table 3 Uni- and multivariate analyses for progression-free and overall survival according for all patients

\begin{tabular}{|c|c|c|c|c|c|c|c|c|}
\hline \multirow[t]{3}{*}{ Variable } & \multicolumn{4}{|c|}{ Progression-free survival } & \multicolumn{4}{|l|}{ Overall survival } \\
\hline & \multicolumn{2}{|l|}{ Univariate } & \multicolumn{2}{|l|}{ Multivariate } & \multicolumn{2}{|l|}{ Univariate } & \multicolumn{2}{|l|}{ Multivariate } \\
\hline & $\mathrm{HR}(95 \% \mathrm{CI})$ & $p$ & $\mathrm{HR}(95 \% \mathrm{CI})$ & $p$ & $\operatorname{HR}(95 \% \mathrm{CI})$ & $p$ & $\operatorname{HR}(95 \% \mathrm{CI})$ & $p$ \\
\hline Age $\geq 70$ vs. $<70$ years & $0.98(0.68-1.42)$ & 0.265 & & & $1.06(0.67-1.67)$ & 0.800 & & \\
\hline Female vs. male & $0.81(0.56-1.17)$ & 0.265 & & & $0.96(0.61-1.51)$ & 0.866 & & \\
\hline ECOG 1 vs. 0 & $0.85(0.56-1.29)$ & 0.441 & $0.81(0.52-1.27)$ & 0.359 & $1.25(0.74-2.10)$ & 0.407 & & \\
\hline ECOG 2 vs. 0 & $1.88(1.00-3.52)$ & 0.049 & $1.01(0.45-2.24)$ & 0.987 & $2.66(1.16-6.11)$ & 0.021 & & \\
\hline ECOG 3 vs. 0 & 7.38 (2.21-24.67) & 0.001 & $7.13(1.65-30.73)$ & 0.008 & $3.51(0.82-15.09)$ & 0.092 & & \\
\hline Therapy line 2 vs. 1 & $1.02(0.65-1.60)$ & 0.941 & & & $0.83(0.47-1.49)$ & 0.534 & $0.83(0.47-1.49)$ & 0.534 \\
\hline Therapy line 3 vs. 1 & $2.00(1.20-3.21)$ & 0.007 & & & $2.00(1.13-3.54)$ & 0.018 & $2.00(1.13-3.54)$ & 0.018 \\
\hline PD-L1 status (neg. vs. $\geq 1 \%$ ) & $1.95(1.30-2.92)$ & 0.001 & $2.04(1.32-3.17)$ & 0.002 & $1.60(0.98-2.61)$ & 0.062 & & \\
\hline Mutational status (pos. vs. neg.) & $1.84(1.09-3.09)$ & 0.023 & & & $1.53(0.80-2.90)$ & 0.198 & & \\
\hline CNS involvement (yes vs. no) & $1.22(0.79-1.89)$ & 0.368 & & & $1.26(0.74-2.16)$ & 0.398 & & \\
\hline Squamous vs. adenocarinoma & $0.88(0.60-1.29)$ & 0.495 & & & $0.93(0.57-1.50)$ & 0.750 & & \\
\hline
\end{tabular}

Figures are given as hazard ratio (95\% confidence interval), with a ratio $>1$ signifying an increased risk of progression/death Bold values are statistically significant $(p<0.05)$

$H R$ hazard ratio, CI confidence interval, ECOG Eastern Cooperative Oncology Group, $P D-L 1$ programmed death-ligand 1, CNS central nervous system

NSCLC patients, of which nearly $50 \%$ were $\geq$ third-line patients. Patients allocated to the placebo group had an OS of 4.7 months and a PFS of 1.8 months [21]. The question arises how our reported $\geq$ third-line collective could have witnessed an obviously longer OS, but a PFS not better than placebo. On the one hand, patients who reached such advanced therapy lines tended to be younger, as mean age decreased with therapy line (72 years in first-, 66 years in second-, and 61 years in $\geq$ third-line patients), whereas ECOG distribution did not show such a trend. On the other hand, the relatively high number of patients with activating alterations in EGFR or ALK in therapy line $\geq 3(N=11$, $29 \%$ ) may have influenced outcomes. Surprisingly, however, we did not find a significant association of either PFS or OS with the presence of that known predictive factor in uniand multivariate analyses. It is likely that patients with such targetable genetic alterations in higher therapy lines may have received less cytotoxic chemotherapies due to available target therapy options and thus still had further and broader treatment options. Of those 11 patients in our collective, $8(73 \%)$ received further therapy, as compared to 11 of 26 (42\%) in the non-mutant patient group. As response rates to ICI mono-therapy in more advanced therapy lines $[3,6$, $15,16]$ as well as in EGFR- or ALK-mutant patients are known to be only modest [12], PFS in $\geq$ third-line therapy is thus expectedly low. However, in our comparably young and frequently EGFR-mutant $\geq$ third-line collective, it seems that a considerable OS could still be attained with subsequent chemo- or target therapies after progression on ICI treatment.
Of interest, the ATLANTIC study on durvalumab evaluated a very similar patient collective of heavily pretreated and frequently EGFR- or ALK-positive patients, and reported results very similar to ours (Table 5) [20]. Also, Lin et al. recently published data on a real-world ICI monotherapy cohort of 74 patients receiving nivolumab or pembrolizumab, of which $41 \%$ were EGFR mutant, $49 \%$ had an ECOG status $\geq 2$, and $69 \%$ received ICI therapy in $\geq$ thirdline therapy. The authors reported an ORR of $32 \%$, PFS was 1.8 , and OS 7.8 months. In a multivariate Cox-proportional hazards analysis, ECOG $\geq 2$ significantly influenced PFS (HR 9.13) and OS (HR 14.72), whereas ICI therapy in $\geq$ third-line therapy did not significantly impact PFS and OS. Analogously to our reported results, Lin et al. found no meaningful influence of EGFR mutation on the cohort outcomes, with a HR of 2.00 ( $p=0.022$-univariate) and 1.26 ( $p=0.534$-multivariate) for PFS and 1.07 for OS (univariate), though there expectedly was a significantly lower treatment response in the EGFR-mutant group with an odds ratio of $0.09(p=0.043)$ [22].

Our study has several limitations. The retrospective design and the relatively small sample size together with numeric differences between the subgroups limited the significance of subgroup analyses. Also, the inclusion criteria may have limited the study results, and a relatively high percentage of stage III patients received first-line monoICI treatment and a few patients also received such therapy due to contraindications to chemotherapy despite a PD-L1 expression $<50 \%$. Another possible limitation is that the re-staging schedule differed slightly between nivolumab 


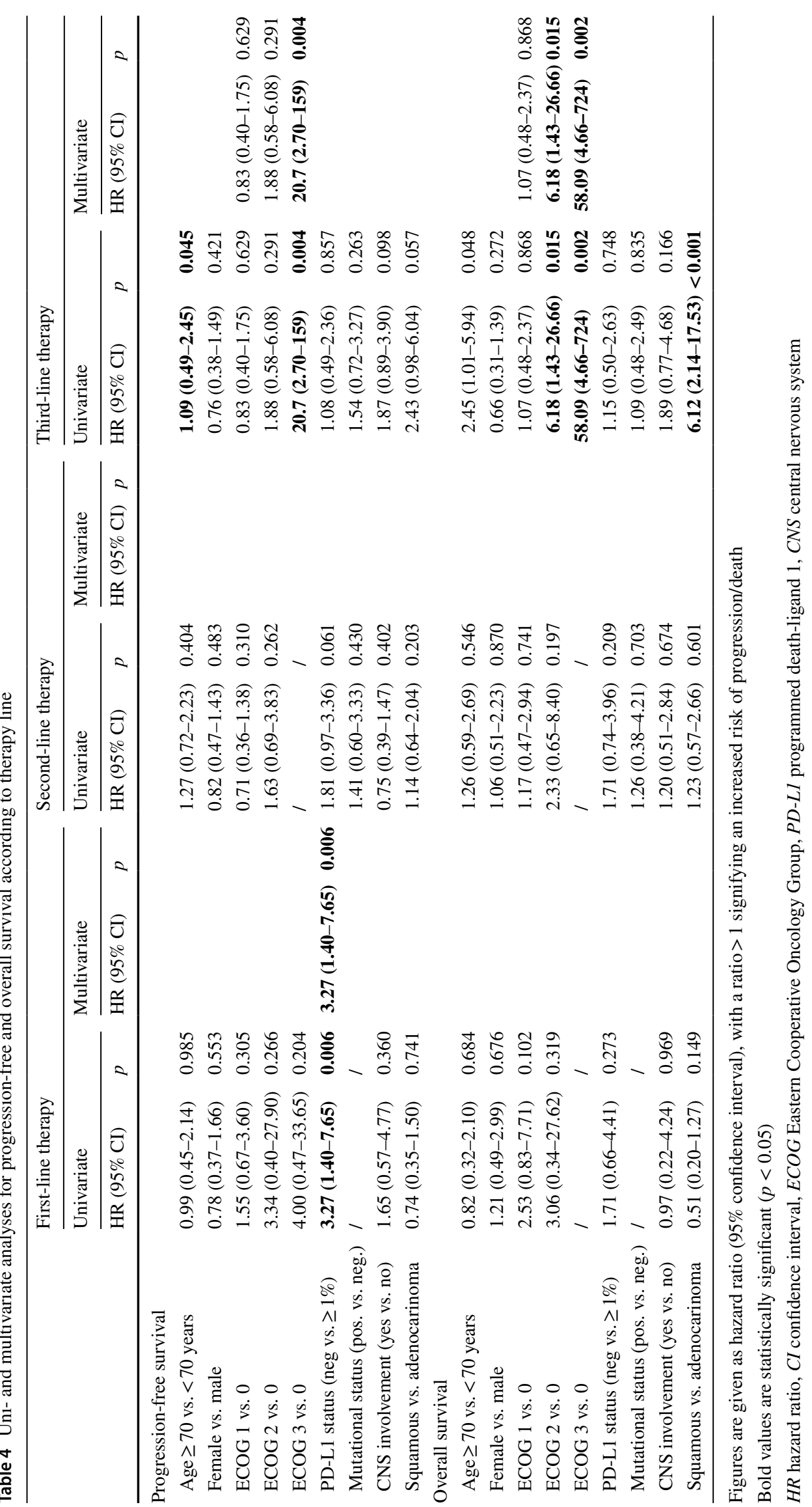




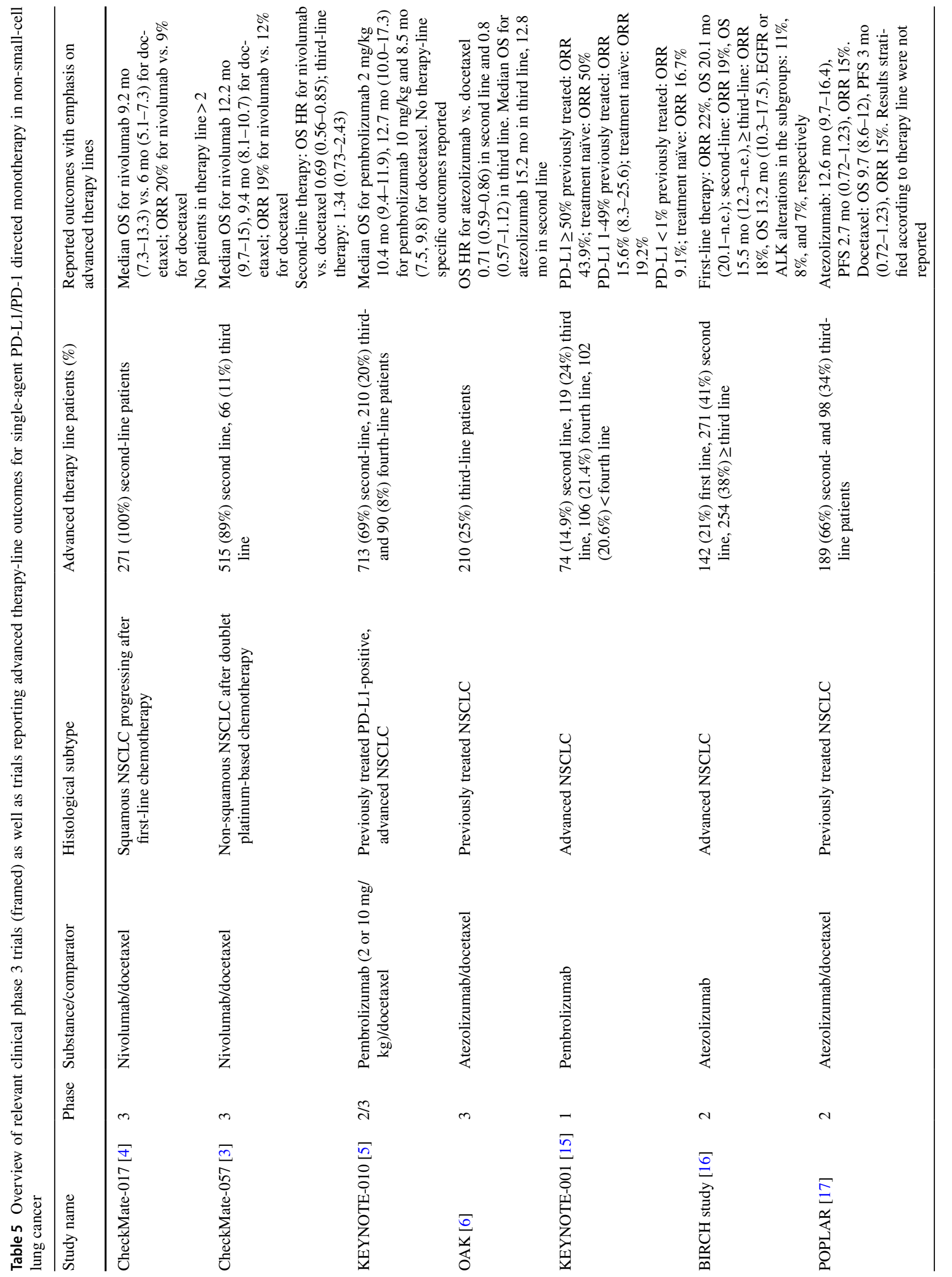




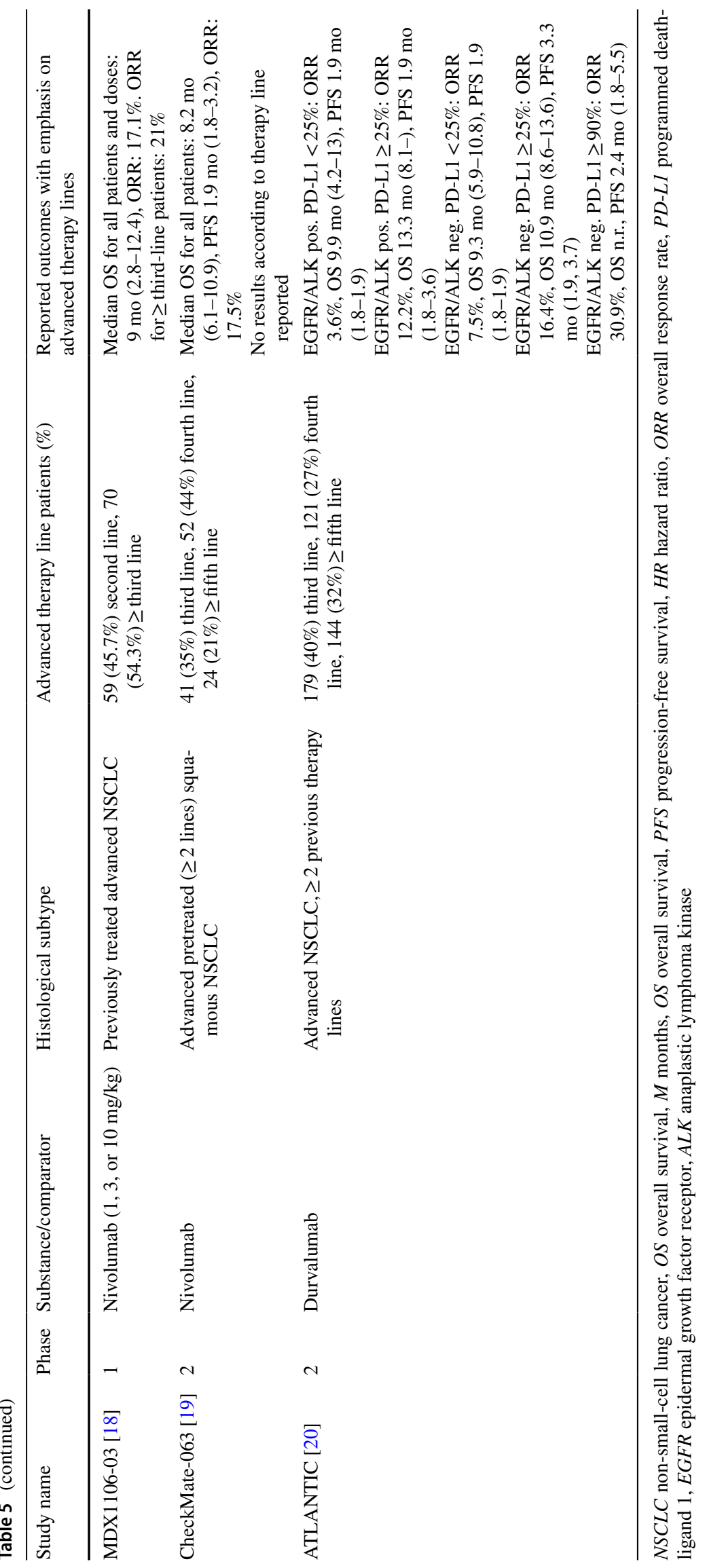


(10 weeks) and pembrolizumab/atezolizumab (12 weeks). As $71 \%$ of patients in $\geq$ third-line treatment received nivolumab, a shorter PFS could partly be explained by that fact. Besides those methodological limitations, the current therapeutic landscape in NSCLC has clearly shifted away from second- or later-line ICI application, as reported in this cohort, to first-line therapy, either in combination with chemotherapy or alone in highly PD-L1-expressing tumors. In line with these recent developments, our reported results support ICI application earlier rather than later in therapy. Still, advances in molecular or clinical characterization of NSCLC patients may again alter the current therapeutic approach, so that our present data could be of value for future considerations.

We conclude that the efficacy of single-agent PD-1/ PD-L1 therapy is lower when applied in more advanced therapy lines $(\geq 3)$. The prognostic value of "traditional" biomarkers like PD-L1 expression or presence of a targetable alteration in EGFR or ALK seems to diminish in third or later therapy lines, where PFS and OS were mainly determined by patient performance status.

Acknowledgements Open access funding provided by Kepler Universitätsklinikum Linz.

\section{Compliance with Ethical Standards}

This study was conducted in accordance with the Declaration of Helsinki and in an entirely retrospective fashion. There was no experimental approach, no additional patient contact, and only patient data assessed in clinical routine were analyzed. Thus, in line with the institutional guidelines, the local ethics committee was not concerned with this study and no written informed consent was obtained from the patients. Patient data were collected in an anonymized fashion and securely electronically stored in a way that only the authors had access to the data. No identifiable patient data have or will ever be published by the authors.

Funding No external funding was used in the preparation of this article.

Conflict of interest DL has received travel/accommodation funding from Roche and Merck Sharp \& Dohme. FH has received travel/accommodation funding from Bristol-Myers Squibb, Roche, and Merck Sharp \& Dohme. GR has served as consultant/advisor to Roche, has received speakers' honoraria from Bristol-Myers Squibb and Roche, and has received travel/accommodation and research funding from Roche. AH has received travel/accommodation funding from Roche. RW has received speakers' honoraria and travel/accommodation funding from and served as consultant/advisor to Roche, Merck Sharp \& Dohme and Bristol-Myers Squibb. EB has received speakers' honoraria from and served as consultant/advisor to Roche, Merck Sharp \& Dohme, and Bristol-Myers Squibb; he has received travel/accommodation funding from Roche and Merck Sharp \& Dohme. GH has served as consultant/advisor to Roche, Merck Sharp \& Dohme, and BristolMyers Squibb. RG has received speakers' honoraria and research funding from Roche, Merck Sharp \& Dohme, and Bristol-Myers Squibb, he has served as consultant/advisor to Roche and Bristol-Myers Squibb, and has received travel/accommodation funding from Roche. BL has received speakers' honoraria from and has served as consultant/advisor to Roche, Merck Sharp \& Dohme, and Bristol-Myers Squibb. KA, MG, and BK declare that they have no conflicts of interest that might be relevant to the contents of this manuscript.

Data availability statement The datasets analyzed during the current study cannot be made publicly available, as they contain possibly identifiable patient data.

Open Access This article is distributed under the terms of the Creative Commons Attribution-NonCommercial 4.0 International License (http://creativecommons.org/licenses/by-nc/4.0/), which permits any noncommercial use, distribution, and reproduction in any medium, provided you give appropriate credit to the original author(s) and the source, provide a link to the Creative Commons license, and indicate if changes were made.

\section{References}

1. Planchard D, Popat S, Kerr K, Novello S, Smit EF, Faivre-Finn $\mathrm{C}$, et al. Metastatic non-small cell lung cancer: ESMO clinical practice guidelines for diagnosis, treatment and follow-up. Ann Oncol. 2018;29:iv192-237.

2. Prelaj A, Tay R, Ferrara R, Chaput N, Besse B, Califano R. Predictive biomarkers of response for immune checkpoint inhibitors in non-small-cell lung cancer. Eur J Cancer. 2019;106:144-59.

3. Borghaei H, Paz-Ares L, Horn L, Spigel DR, Steins M, Ready NE, et al. Nivolumab versus docetaxel in advanced nonsquamous non-small-cell lung cancer. N Engl J Med. 2015;373:1627-39.

4. Brahmer J, Reckamp KL, Baas P, Crinò L, Eberhardt WEE, Poddubskaya E, et al. Nivolumab versus docetaxel in advanced squamous-cell non-small-cell lung cancer. N Engl J Med. 2015;373:123-35.

5. Herbst RS, Baas P, Kim D-W, Felip E, Pérez-Gracia JL, Han J-Y, et al. Pembrolizumab versus docetaxel for previously treated, PD-L1-positive, advanced non-small-cell lung cancer (KEYNOTE-010): a randomised controlled trial. Lancet. 2016;387:1540-50.

6. Rittmeyer A, Barlesi F, Waterkamp D, Park K, Ciardiello F, von Pawel J, et al. Atezolizumab versus docetaxel in patients with previously treated non-small-cell lung cancer (OAK): a phase 3 , open-label, multicentre randomised controlled trial. Lancet. 2017;389:255-65.

7. Reck M, Rodríguez-Abreu D, Robinson AG, Hui R, Csőszi T, Fülöp A, et al. Pembrolizumab versus chemotherapy for PD-L1-positive non-small-cell lung cancer. N Engl J Med. 2016;375:1823-33.

8. Gandhi L, Rodríguez-Abreu D, Gadgeel S, Esteban E, Felip E, De Angelis F, et al. Pembrolizumab plus chemotherapy in metastatic non-small-cell lung cancer. N Engl J Med. 2018;378:2078-92.

9. Paz-Ares L, Luft A, Vicente D, Tafreshi A, Gümüş M, Mazières J, et al. Pembrolizumab plus chemotherapy for squamous non-smallcell lung cancer. N Engl J Med. 2018;379:2040-51.

10. Socinski MA, Jotte RM, Cappuzzo F, Orlandi F, Stroyakovskiy $\mathrm{D}$, Nogami N, et al. Atezolizumab for first-line treatment of metastatic nonsquamous NSCLC. N Engl J Med. 2018;378:2288-301.

11. Hellmann MD, Ciuleanu T-E, Pluzanski A, Lee JS, Otterson GA, Audigier-Valette C, et al. Nivolumab plus ipilimumab in lung cancer with a high tumor mutational burden. N Engl J Med. 2018;378:2093-104.

12. Lee CK, Man J, Lord S, Cooper W, Links M, Gebski V, et al. Clinical and molecular characteristics associated with survival 
among patients treated with checkpoint inhibitors for advanced non-small cell lung carcinoma. JAMA Oncol. 2018;4:210.

13. Chansky K, Detterbeck FC, Nicholson AG, Rusch VW, Vallières $\mathrm{E}$, Groome P, et al. The IASLC lung cancer staging project: external validation of the revision of the TNM stage groupings in the eighth edition of the TNM classification of lung Cancer. J Thorac Oncol. 2017;12:1109-1121.

14. Eisenhauer EA, Therasse P, Bogaerts J, Schwartz LH, Sargent D, Ford R, et al. New response evaluation criteria in solid tumours: revised RECIST guideline (version 1.1). Eur J Cancer. 2009;45:228-47.

15. Garon EB, Rizvi NA, Hui R, Leighl N, Balmanoukian AS, Eder JP, et al. Pembrolizumab for the treatment of non-small-cell lung cancer. N Engl J Med. 2015;372:2018-28.

16. Peters S, Gettinger S, Johnson ML, Jänne PA, Garassino MC, Christoph D, et al. Phase II trial of atezolizumab as first-line or subsequent therapy for patients with programmed death-ligand 1-selected advanced non-small-cell lung cancer (BIRCH). J Clin Oncol. 2017;35:2781-9.

17. Fehrenbacher L, Spira A, Ballinger M, Kowanetz M, Vansteenkiste J, Mazieres J, et al. Atezolizumab versus docetaxel for patients with previously treated non-small-cell lung cancer (POPLAR): a multicentre, open-label, phase 2 randomised controlled trial. Lancet. 2016;387:1837-46.
18. Gettinger SN, Horn L, Gandhi L, Spigel DR, Antonia SJ, Rizvi NA, et al. Overall survival and long-term safety of nivolumab (anti-programmed death 1 antibody, BMS-936558, ONO-4538) in patients with previously treated advanced non-small-cell lung cancer. J Clin Oncol. 2015;33:2004-12.

19. Rizvi NA, Mazières J, Planchard D, Stinchcombe TE, Dy GK, Antonia SJ, et al. Activity and safety of nivolumab, an anti-PD-1 immune checkpoint inhibitor, for patients with advanced, refractory squamous non-small-cell lung cancer (CheckMate 063): a phase 2, single-arm trial. Lancet Oncol. 2015;16:257-65.

20. Garassino MC, Cho B-C, Kim J-H, Mazières J, Vansteenkiste $\mathrm{J}$, Lena $\mathrm{H}$, et al. Durvalumab as third-line or later treatment for advanced non-small-cell lung cancer (ATLANTIC): an openlabel, single-arm, phase 2 study. Lancet Oncol. 2018;19:521-36.

21. Shepherd FA, Rodrigues Pereira J, Ciuleanu T, Tan EH, Hirsh V, Thongprasert S, et al. Erlotinib in previously treated non-smallcell lung cancer. N Engl J Med. 2005;353:123-32.

22. Lin S-Y, Yang C-Y, Liao B-C, Ho C-C, Liao W-Y, Chen K-Y, et al. Tumor PD-L1 expression and clinical outcomes in advanced-stage non-small cell lung cancer patients treated with nivolumab or pembrolizumab: real-world data in Taiwan. J Cancer. 2018;9:1813-20.

\section{Affiliations}

\section{David Lang ${ }^{1} \mathbb{1}$. Florian Huemer ${ }^{2}$. Gabriel Rinnerthaler ${ }^{2} \cdot$ Andreas Horner $^{1} \cdot$ Romana Wass $^{1} \cdot$ Elmar Brehm $^{1}$. Kaveh Akbari ${ }^{3} \cdot$ Marcel Granitz $^{4} \cdot$ Georg Hutarew ${ }^{5} \cdot$ Bernhard Kaiser $^{1} \cdot$ Richard Greil $^{2} \cdot$ Bernd Lamprecht $^{1}$}

Florian Huemer

onkologie.salzburg@ @alk.at

Kaveh Akbari

radiologie@kepleruniklinikum.at

Marcel Granitz

radiologie@salk.at

Georg Hutarew

pathologie@salk.at

1 Department of Pulmonology, Kepler University Hospital Linz, Krankenhausstrasse 9, 4020 Linz, Austria

2 Department of Internal Medicine III with Haematology, Medical Oncology, Haemostaseology, Infectiology and Rheumatology, Oncologic Center, Salzburg Cancer Research Institute, Laboratory for Immunological and Molecular Cancer Research (SCRI-LIMCR), Paracelsus Medical University, Cancer Cluster Salzburg, Muellner Hauptstrasse 48, 5020 Salzburg, Austria

3 Central Radiology Institute, Kepler University Hospital, Krankenhausstrasse 9, 4020 Linz, Austria

4 Institute of Diagnostic Radiology, Paracelsus Medical University Salzburg, Muellner Hauptstrasse 48, 5020 Salzburg, Austria

5 Institute of Pathology, Paracelsus Medical University Salzburg, Muellner Hauptstrasse 48, 5020 Salzburg, Austria 